\title{
The Analysis of Residual Contaminants and Migration of Leakage at Pore Scale in Leakage Process of Pipeline
}

\author{
Haiying Qi \\ D\&P Technology Institute, LiaoHe Oil Field Company, Petrochina Panjin, Liaoning Province, \\ PRC, 124010 \\ lhzdx@163com
}

Keywords: Buried Pipeline Leakage; Pore Scale; Crude Oil; Residual Model

Abstract The buried pipeline leakage has tremendous impact on the surrounding environment, while crude oil migration and residues of contaminants in the soil porous media law is an important basis for the detection and elimination Considering the characteristics of oil multiphase flow in porous media, we establish underground pipeline leak oil contaminants diffusion model under pore-scale to analyze the influence of the porous medium particle size, pore structure, and the nature on its oil migration and the residues The results show that Plasmid size has the more influences on the oil residues in the soil porous media, light crude oil varying with time gradually migrate out from small gap between the ball, stop separating from the water after 20 seconds, some part of light crude oil still adhere to the wall and near the ball

\section{Introduction}

Oil leakage of Pipelines often caused by corrosion or breaking during the operation of oil gathering pipeline, which cause serious pollution to the soil and surrounding environment, so studying the characteristics of pipeline leakage contaminant residues is of great significance to the later environmental restoration The domestically study mainly focuses on the simulation of influence of leakage position on leakage rate, the relationship between the leakage rate and leakage depth, the relationship between the speed and depth of the leakage It has been demonstrated that the leakage diffusion and oil pollutant in groundwater which is obtained and the leaked oil adhesion in the riverbed will be augmented with the increase of groundwater flow velocity, leakage mouth in the upper part pipeline minimum leakage range, and conclusions such as landmark diffusion area is the most popular ${ }^{[1-4]}$ Factors such as the pore structure and leakage velocity have been controlled in this article The migration of pollutants is simulated after pipeline leakage.

\section{Mathematical model}

The buried pipelines leaked pollutants is porous flow medium in this article the soil particles are simplified as the radius of 3-4 mm balls, the space between the ball all assumed to be the air, according to the leakage process and selection of boundary conditions, this article uses the VOF model and establishes conservation of mass and momentum conservation equation

\section{Mass conservation equation}

$$
\frac{\partial}{\partial t}\left(\rho_{m}\right)+\nabla \cdot\left(\rho_{m} v_{m}\right)=0
$$

In the formula:

$\mathrm{m}$ - The number of multiphase fluid.

$v_{m}$-The average velocity of fluid, $\mathrm{m} / \mathrm{s}$.

$$
v_{m}=\frac{\sum_{k=1}^{m} \alpha_{k} \rho_{k} v_{k}}{\rho_{m}}
$$


$\rho_{m}$-The density of multiphase fluid, $\mathrm{kg} / \mathrm{m}^{3}$.

$$
v_{m}=\frac{\sum_{k=1}^{m} \alpha_{k} \rho_{k} v_{k}}{\rho_{m}}
$$

$\alpha_{k}$-The volume ratio in the k's phase.

$v_{k}$-The velocity in the $\mathrm{k}$ 's phase, $\mathrm{m} / \mathrm{s}$.

$\rho_{k}$-The density in the k's phase, $\mathrm{kg} / \mathrm{m}^{3}$.

\section{The momentum conservation equation}

$$
\frac{\partial}{\partial t}\left(\rho_{m}\right)+\nabla \cdot\left(\rho_{m} v_{m}\right)=-\nabla \cdot\left[\mu_{m}\left(\nabla v_{m}+\nabla v_{m}^{T}\right)\right]+\rho_{m} g+F+\nabla \cdot\left(\sum_{k=1}^{n} \alpha_{k} \rho_{k} v_{d r, k}\right)
$$

In the formula:

$\mathrm{m}$-The number of multiphase fluid.

$F$-Volume force, N.

$\nabla p$ - The flowing differential pressure, $\mathrm{Pa}$.

$\mu_{m}$-The viscosity of the multiphase fluid, $\mathrm{kg} / \mathrm{m} \cdot \mathrm{s}$.

$$
\mu_{m}=\sum_{k=1}^{n} \alpha_{k} \mu_{k}
$$

$v_{d r, k}$-Drive speed in the k's phase relative to the multiphase fluid, it is $v_{d r, k}=v_{k}-v_{m}, \mathrm{~m} / \mathrm{s}$.

\section{Simulation conditions}

Select VOF model, due to the simulation boundary dimensions, the small ball size, making the porosity uniform use 0569 , it is concluded that the viscous resistance coefficient is 4201521 , the co inertial resistance coefficient is 1364763 the size of the spill area is $300 \times 300 \mathrm{~mm}$, the diameter of leakage pipe is $20 \mathrm{~mm}$, the size of leakage mouth is $7 \mathrm{~mm}$.

The numerical simulation has been performed under pore scale Particle radius of $3 \mathrm{~mm}$ and $4 \mathrm{~mm}$ is selected, respectively Arrangement mode is the straight and alternating forms The size of the simulation area is $300 \times 300 \mathrm{~mm}$, the diameter of leakage pipe is $20 \mathrm{~mm}$, the size of leakage mouth is $7 \mathrm{~mm}$, the porosity of porous medium is 0569 .

The use of triangle mesh of porous media model, the grid size is 05 pore scale grid partition uses two kinds of particle radius with $3 \mathrm{~mm}$ and $4 \mathrm{~mm}$, respectively and use the straight and horizontal line to draw in two ways, for the triangular grid, size of 05 local grid diagram as shown in figure 1

The grid number in table 1

\section{Table 1}

\begin{tabular}{|c|c|c|}
\hline Arrangement & The straight line & Alternately \\
\hline Particle radius & 446584 & 442300 \\
\hline $4 \mathrm{~mm}$ & 437668 & 439178 \\
\hline
\end{tabular}

Leakage area boundary for the pressure of 0 pa's export, leakage mouth entrance for speed, the speed is $011 \mathrm{~m} / \mathrm{s}$ considering the influence of gravity, acceleration of gravity is $981 \mathrm{~m} / \mathrm{s}^{2}$, the particle surface is wall, the ratio of oil and water is $1: 1$.

The material for oil-water two phase simulation use water and decane $(\mathrm{C} 10 \mathrm{H} 22)$, main properties can be seen in the table below. 
Table 2

\begin{tabular}{|c|c|c|c|}
\hline Property & water & oil & $\begin{array}{c}\text { Oil and water, on } \\
\text { average }\end{array}$ \\
\hline Density & $9982 \mathrm{~kg} / \mathrm{m}^{3}$ & $730 \mathrm{~kg} / \mathrm{m}^{3}$ & $8641 \mathrm{~kg} / \mathrm{m}^{3}$ \\
\hline Viscosity & $000103 \mathrm{~Pa} \cdot \mathrm{s}$ & $00024 \mathrm{~Pa} \cdot \mathrm{s}$ & $0001715 \mathrm{~Pa} \cdot \mathrm{s}$ \\
\hline
\end{tabular}

The influence of particle size

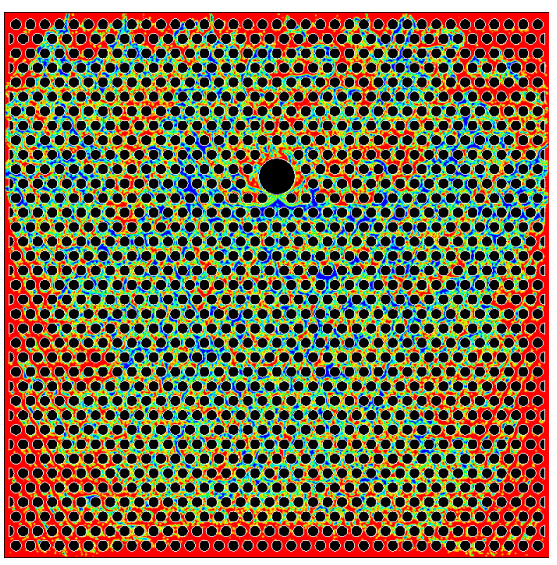

a

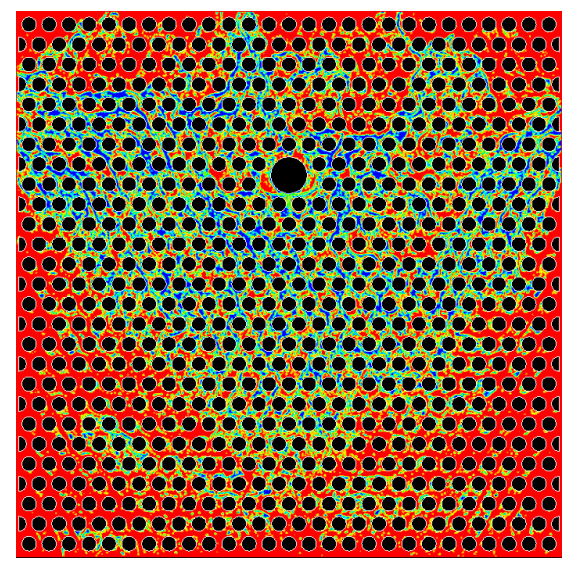

$\mathrm{b}$

Under the $3 \mathrm{~mm}$ diameter oil phase distribution before the leakage mouth shut down(a)

Under the $4 \mathrm{~mm}$ diameter oil phase distribution before the leakage mouth shut down(b)

Fig1. The oil-water mixture phase diagram under the different particle diameter before the leakage mouth shut down

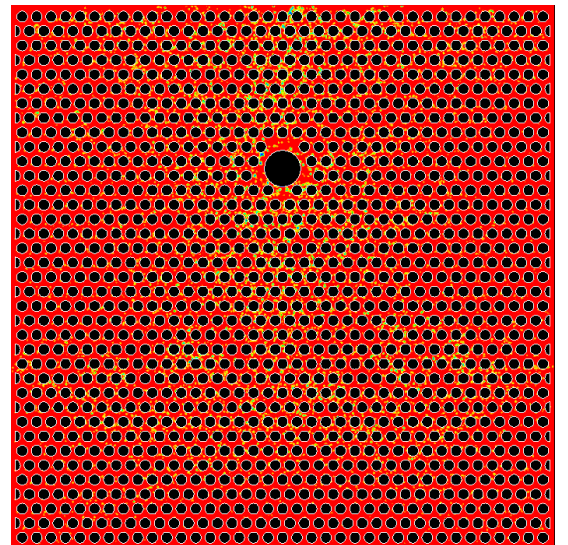

a

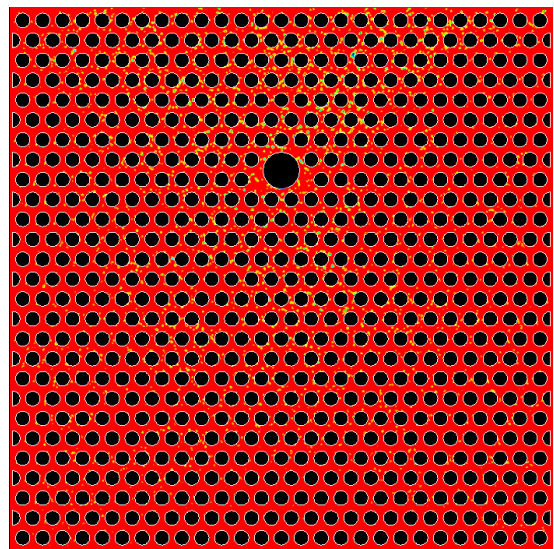

$\mathrm{b}$

Under the $3 \mathrm{~mm}$ diameter oil phase distribution after the leakage mouth shut down $15 \mathrm{~s}(\mathrm{a})$

Under the $4 \mathrm{~mm}$ diameter oil phase distribution after the leakage mouth shut down $15 \mathrm{~s}(\mathrm{~b})$

Fig 2. The oil-water mixture phase diagram under the different particle diameter after the leakage mouth shut down

As you can see from figure 1, in the same flow rate, the condition of small ball diameter size will impact on mixed pollutants leakage distribution, when particle size large, light oil concentrates distribution in the upper, diffusion range is small. Particle size small, lightweight oil dispersed evenly, wide spread.

As you can see from figure 2 , light oil were separated with water after $15 \mathrm{~s}$, migrate to outside surface on the simulation range, but a few size small residual is near the leak. 


\section{Export density changes over time}

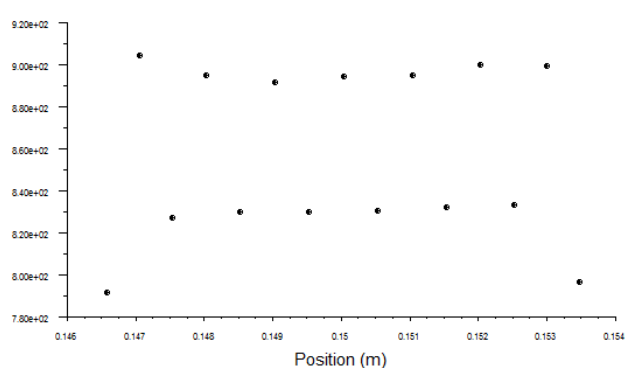

$4 \mathrm{~S}$ outlet flow density graph a

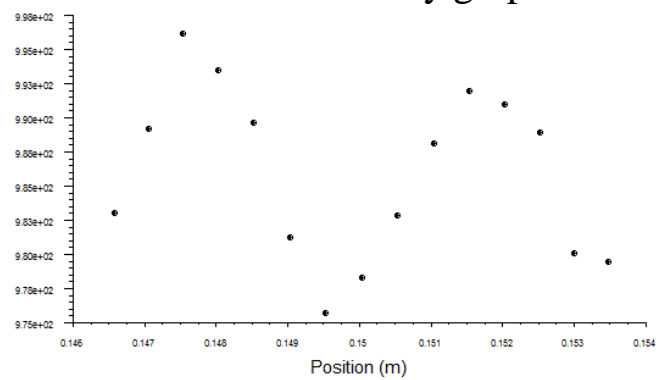

23S outlet density graph c

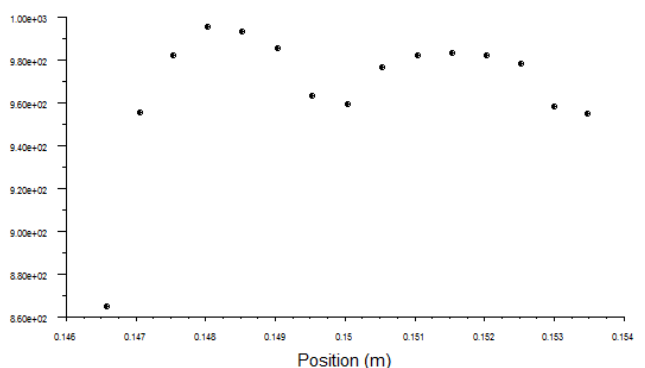

$7.5 \mathrm{~S}$ outlet density graph $\mathrm{b}$

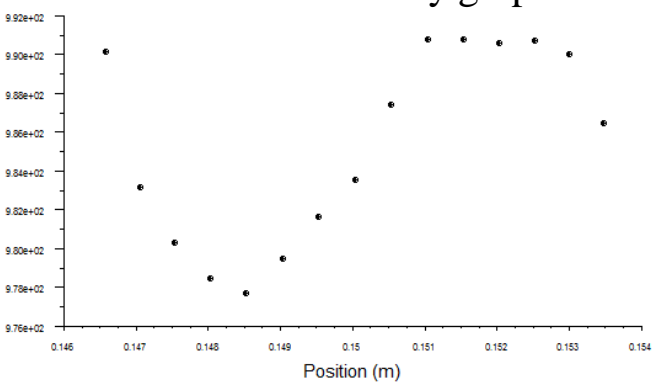

40S outlet density graph d

Fig 3.The $4 \mathrm{~mm}$ diameter near the leakage mouth density variation over time

Close the leakage after mouth, leakage near the mouth density decreases with time which can know from figure 3, After $20 \mathrm{~s}$ density almost no changes, but still uneven density, may safely draw the conclusion: Light crude which changes gradually over time migration out from the space between the ball, from the water after $20 \mathrm{~s}$ almost stop separation process, there is still some light crude adhesion in the tube wall and near the ball.

\section{Conclusions}

In this paper, residual characteristics of buried oil pipeline leak pollutants are simulated with the CFD software, contrast analysis before and after the leakage from the CFD simulation, it can be conclueded that:

(1) Small ball diameter size will impact on mixed pollutants leakage distribution, the greater particle size, the easier it is upward migration, and the light oil residue is less.

(2) Light crude which changes gradually with time from the space between the ball can migrate out, almost stop separating from the water after 20s, there is still some light crude adhered on the tube wall and near the ball.

\section{References}

[1] Shi Long, Ma Guiyang et. The Numerical Study on Crude Oil Leakage of Underwater Buried Pipeline [J]. Contemporary Chemical Industry(In Chinese), 2014,43(1):153-155.

[2] Ma Yue, Wang Yue. Simulation Analysis of the Range of Soil Spread by Buried Pipeline Leakage [J]. Journal of Beijing Institute of Petro-chemical Technology(In Chinese), 2013, 20(4): 24-28.

[3] Li Zhaoyang, Ma Guiyang, Liu Liang. Diffusion simulation on leaked oil for buried pipelines[J]. Oil and Gas Storage and Transportation(In Chinese), 2011, 30(9): 674-676.

[4] Gao Xue0li, Ma Gui-yang.Numerical Simulation on Speepage of Underground Pipeline Leakage[J]. Journal of Liaoning Petrochemical University(In Chinese), 2011,31(2):20-23. 